\title{
Consumo alimentar e gasto energético em adolescentes obesos e eutróficos
}

\author{
Food consumption and energy expenditure in obese and non-obese adolescents
}

\author{
Camila Dayrell ${ }^{1}$, Regina Urasaki ${ }^{2}$, Rita Maria M. Goulart ${ }^{3}$, Sandra Maria L. Ribeiro ${ }^{4}$
}

\section{RESUMO}

Objetivo: Comparar, entre adolescentes obesos e eutróficos, variáveis relacionadas ao consumo alimentar e ao gasto energético.

Métodos: Estudo transversal com 23 adolescentes recrutados em escolas e centros de recreação de São Paulo (SP), distribuídos em dois grupos: 11 obesos e 12 eutróficos. Foram avaliados: peso corporal, estatura, índice de massa corporal, circunferência da cintura, massa magra e massa gorda (estimados por bioimpedância elétrica), três recordatórios alimentares de 24 horas, estimativa do gasto energético diário a partir de três métodos (recordatórios de atividade física, fórmulas preditivas e calorimetria indireta), gasto energético de repouso e a potência aeróbia.

Resultados: O gasto energético diário dos obesos foi maior, porém a ingestão alimentar se assemelhou à dos eutróficos. Não foram encontradas diferenças no gasto energético de repouso entre os grupos, porém a potência aeróbia foi maior nos eutróficos. A dieta de ambos os grupos não foi considerada saudável, mas os obesos apresentaram menor consumo de vários alimentos considerados saudáveis, como hortaliças. Observou-se discrepância na análise do gasto energético diário, quando se adotaram diferentes métodos.

Conclusões: Os adolescentes apresentaram padrão alimentar associado ao risco de obesidade e doenças crônicas. Mais estudos são necessários para identificar melhores métodos para avaliar seu gasto energético. É urgente a busca de estratégias de educação nutricional específicas para esse grupo etário.

Palavras-chave: adolescente; consumo de alimentos; avaliação nutricional; metabolismo energético; obesidade.

\section{ABSTRACT}

Objective: To compare food consumption and energy expenditure between obese and non-obese adolescents.

Methods: This cross-sectional study comprised 23 adolescents recruited from schools and recreation centers from São Paulo (SP), Brazil, distributed in two groups: 11 obese and 12 non-obese. The following variables were analyzed: body weight, height, body mass index, waist circumference, fat and lean mass (estimated from bioelectrical impedance), three days' 24-hour food recall, daily energy expenditure from three different methods (three days' physical activity recall, predictive equations and indirect calorimetry), resting energy expenditure and aerobic power test.

Results: Daily energy expenditure was higher in the obese group, but food ingestion presented no difference between groups. There was no difference in resting energy expenditure between obese and non-obese groups and aerobic power was higher in non-obese group. Neither obese nor non-obese adolescents presented adequate feeding behavior; however, the obese group presented lower consumption of healthy foods like vegetables. There was disagreement among different methods of analysis of daily energy expenditure.

Conclusions: All adolescents presented feeding behaviors related to risk for chronic diseases. New studies are necessary to identify the best way to analyze energy expenditure. Nutritional education strategies directed towards adolescents are needed.

Key-words: adolescent; food consumption; nutrition assessment; energy metabolism; obesity.
Instituição: Universidade São Judas Tadeu (USJT), São Paulo, SP, Brasil ${ }^{1}$ Bacharel em Nutrição pela USJT, São Paulo, SP, Brasil

${ }^{2}$ Educadora Física; Mestre em Educação Física pela USJT, São Paulo, SP, Brasil

${ }^{3}$ Doutora em Saúde Pública pela Faculdade de Saúde Pública da Universidade de São Paulo (USP); docente do Curso de Nutrição da USJT, São Paulo, SP, Brasil

${ }^{4}$ Doutora em Nutrição Humana Aplicada pela USP; docente da Escola de Artes, Ciências e Humanidades da USP; colaboradora do Programa de Mestrado em Educação Física da USJT, São Paulo, SP, Brasil
Endereço para correspondência:

Sandra Maria Lima Ribeiro

Avenida Arlindo Bettio, 1000 - Ermelino Matarazzo

CEP 03828-000 - São Paulo/SP

E-mail: smlribeiro@usp.br

Recebido em: 7/2/09

Aprovado em: 11/6/09 


\section{Introdução}

Vários fatores interferem no consumo alimentar de adolescentes, tais como preocupações com a imagem corporal, convivência social, influência da mídia e maior período fora de casa, entre outros. Os adolescentes são considerados vulneráveis do ponto de vista nutricional devido ao padrão dietético tipicamente baixo em micronutrientes e alto em açúcares simples e gorduras saturadas. Esses fatores predispõem ao aumento da gordura corporal, culminando em obesidade e em maior risco para o desenvolvimento de doenças ${ }^{(1)}$.

A obesidade é uma epidemia que atinge todos os níveis socioeconômicos, tanto nos países desenvolvidos quanto nos em desenvolvimento. No Brasil, sua prevalência em jovens aumentou 8,9\% no período de 1974-1997(2). É uma doença de origem multifatorial, abrangendo fatores genéticos, metabólicos, fisiológicos ou ainda relacionados ao estilo de vida. No que diz respeito a aspectos fisiológicos e metabólicos, estudos têm procurado identificar se o gasto energético em repouso ou em resposta à prática de atividade física está reduzido em obesos, colaborando com o ganho de peso corporal ${ }^{(3)}$.

Considerando o aumento da incidência de obesidade em adolescentes, é importante que se conheça com mais profundidade alguns fatores associados a esse desvio nutricional. Adolescentes obesos apresentam padrões alimentares diferentes de adolescentes não-obesos? Uma hipótese a ser levantada nesse sentido é a de que adolescentes obesos apresentam pior padrão alimentar. Ainda, adolescentes obesos demonstram diferentes padrões de gasto energético no repouso ou em resposta à atividade física? Imagina-se que os diferentes componentes do gasto energético são fatores que podem explicar, pelo menos em parte, a obesidade. Dessa forma, este estudo teve o objetivo de comparar, entre adolescentes obesos e eutróficos, algumas variáveis relacionadas ao consumo alimentar e ao gasto energético. Pretende-se, assim, colaborar com o desenvolvimento de programas alimentares e de atividade física para esses indivíduos.

\section{Métodos}

O recrutamento dos sujeitos foi realizado em escolas e centros de recreação para adolescentes na cidade de São Paulo (SP), no período de março a maio de 2007. A partir desse contato, os indivíduos que se voluntariaram foram investigados quanto aos critérios de inclusão e exclusão. Foram considerados critérios de inclusão: sexo masculino, idade de
15 a 18 anos, estágio de desenvolvimento puberal caracterizado como pós-púbere identificado pela Prancha de Tanner ${ }^{(4)}$, nível de atividade física entre moderado e alto, identificado por meio do Questionário Internacional de Atividade Física $(\text { IPAQ })^{(5)}$. Os critérios de exclusão foram ingestão de algum medicamento que pudesse interferir na taxa metabólica ou ainda algum tipo de restrição à prática de exercícios físicos. Trata-se, portanto, de uma amostra não-probabilística e de conveniência.

Todos os responsáveis pelos voluntários, após tomarem ciência dos procedimentos, assinaram um Termo de Consentimento e o projeto foi aprovado pelo Comitê de Ética da Universidade São Judas Tadeu (USJT). Os procedimentos de avaliação foram realizados em três dias não-consecutivos, sendo detalhados a seguir:

- Dia 1: os adolescentes foram distribuídos em grupos experimentais (obesos, ou OB, e eutróficos, ou EU) e esclarecidos quanto aos procedimentos. Foram avaliados: peso corporal $\left(\right.$ Filizola $\left.^{\circledR}\right)$, estatura $\left(\right.$ Secca $\left.^{\circledR}\right)$ e índice de massa corporal (IMC, equivalente a peso/estatura ${ }^{2}$ ). O IMC foi classificado a partir de Must et a $l^{(6)}$ e da classificação da Organização Mundial de Saúde ${ }^{(7)}$. Foram aplicados os primeiros recordatórios de 24 horas - de alimentação e de atividade física;

- Dia 2: os adolescentes foram previamente orientados a chegar ao laboratório entre 8 e 9h, após uma noite de sono e jejum de oito horas, sem atividade física intensa nas últimas 24 horas. Foi mensurada a circunferência da cintura (CC), no ponto médio entre a crista ilíaca e a última costela. Foi adotado o percentil 80 (P80) como ponto de corte para identificação do risco de doenças crônicas ${ }^{(8)}$. A massa magra corporal (MMC) e massa gorda corporal (MGC) foram estimadas por bioimpedância tetrapolar (Biodynamics ${ }^{\circledR} 450$ ), utilizando-se equações específicas para a faixa etária. É importante destacar que não existe método para análise da composição corporal em obesos isento de limitações e críticas ${ }^{(9)}$. Para classificar a MMC e a MGC, utilizaram-se os valores propostos por Pichard et a ${ }^{(10)}$. Avaliou-se o gasto energético de repouso (GER) por calorimetria indireta (VO2000, INBRASPORT ${ }^{\circledR} \mathrm{e}$ software AEROGRAF ${ }^{\circledR}$ ). Os adolescentes foram acomodados em uma maca, em ambiente termicamente estável, com iluminação e ruídos controlados, sendo acoplada ao rosto máscara para analisar a troca gasosa. Os indivíduos permaneceram em repouso por 30 minutos: os primeiros 15 minutos para adaptação ao equipamento e os $15 \mathrm{mi}-$ nutos finais para o cálculo propriamente dito. O segundo 
recordatório de 24 horas para alimentação e atividade física foi aplicado;

- Dia 3: foi realizado o teste de potência aeróbia. Os adolescentes foram orientados a não realizarem exercício físico intenso nas 24 horas anteriores, a se alimentarem com refeição leve aproximadamente duas horas antes do teste, a não ingerirem nenhum alimento que contivesse cafeína e a trajarem roupas leves. Antes, durante e depois do teste, foram mensuradas a pressão arterial (PA - esfignomanômetro aneróide Sanny ${ }^{\circledR}$ ) e a frequência cardíaca (FC - frequêncímetro Polar ${ }^{\circledR}$, modelo S810). O monitoramento do ritmo cardíaco e das condições do miocárdio antes, durante e após o teste foi realizado por eletrocardiograma $\left(\right.$ Cardio $\left.\mathrm{Control}^{\oplus}\right)$. Para o teste propriamente dito, o protocolo foi do tipo rampa, com potência inicial de $30 \mathrm{~W}$ e incremento de $15 \mathrm{~W}$ a cada minuto, mantendo-se 60 rotações por minuto. O teste foi executado até a fadiga referida pelos indivíduos. Aplicaram-se, nesse dia, os terceiros recordatórios (alimentar e de atividade física).

Os recordatórios alimentares foram analisados quanto ao valor energético e à distribuição em macronutrientes, com o auxílio do software NUT-WIN ${ }^{\circledR}$ e confrontados com as referências de ingestão (dietary reference intakes, ou DRIs ${ }^{(11)}$ ). Foi determinado o consumo de porções diárias dos diferentes grupos de alimentos propostos pelo Guia Alimentar da População Brasileira ${ }^{(12)}$. Observou-se a variedade da dieta ${ }^{(13)}$, a realização diária do café da manhã, o consumo de refrigerantes, além do número de refeições diárias. Considerou-se 'refeição' todo o momento em que fossem ingeridos alimentos fontes de nutrientes essenciais ${ }^{(14)}$.

Os recordatórios de atividade física foram preenchidos pelos avaliadores em intervalos de 15 minutos e de acordo com a intensidade percebida (sendo 1 equivalente ao repouso e 9 à atividade muito intensa). Essas informações foram avaliadas de acordo com o valor em unidades metabólicas (METs), convertidas em calorias gastas. A somatória de toda a energia gasta no dia correspondeu ao gasto energético diário $^{(15)}$. Tanto os recordatórios alimentares quanto os de atividade física foram calculados tomando por base a média dos três dias analisados.

O gasto energético diário (GED) foi avaliado de três diferentes maneiras: GED1, a partir do cálculo dos recordatórios de atividade física, como descrito acima; GED2, pelas equações preditivas e pelos múltiplos referentes à atividade física propostas pela Food and Agriculture Organization (FAO),
World Health Organization (WHO) e United Nations University (UNU) ${ }^{(16)}(10-17$ anos $=17,5$ xpeso $+651 ; 18-29$ anos $=15,3$ xpeso +679 ; fator atividade moderado: 1,6 ; fator atividade alto: 1,9); e GED3, por calorimetria indireta. Para o cálculo do gasto energético de repouso, foi empregada a fórmula de Jéquier e Schultz ${ }^{(17)}$ :

$$
\mathrm{GER}=\left[(4,686+1,096 \mathrm{x}(\mathrm{QR}-0,707)) \mathrm{x} \mathrm{VO}_{2}\right] \mathrm{x} 24
$$

onde: $\mathrm{QR}=$ quociente respiratório e $\mathrm{VO}_{2}=$ consumo de oxigênio em $\mathrm{mL} \cdot \mathrm{kg}^{-1} \cdot \mathrm{min}^{-1}$.

Os valores obtidos foram multiplicados pelos múltiplos referentes à atividade física ${ }^{(16)}$.

Os dados foram analisados quanto à distribuição (teste de Shapiro-Wilk) e apresentados sob a forma de média e desvio padrão (dados com distribuição normal) ou de mediana, valores mínimos e máximos (dados com distribuição não normal). Foram adotados testes paramétricos (teste $t \mathrm{de}$ Student para amostras independentes, teste $t$ para amostras dependentes e coeficiente de correlação de Pearson) ou nãoparamétricos (teste U de Mann Whitney e coeficiente de correlação de Spearman). Foi fixado o nível de significância de $5 \%(p \leq 0,05)$. O software estatístico utilizado foi o Statística $^{\circledR}$, versão 7.0.

\section{Resultados}

Foram estudados 11 adolescentes eutróficos (EU: $16,8 \pm 1,1$ anos) e 12 obesos (OB: 15,5 $\pm 0,8$ anos). Os valores antropométricos, de composição corporal e de consumo de oxigênio estão apresentados na Tabela 1. Não houve diferença entre os grupos no GER e o grupo EU apresentou maior $\mathrm{VO}_{\text {2pico }}$ Quando avaliado em valores absolutos (kcal.dia ${ }^{-1}$, dados não exibidos na Tabela 1), o gasto energético de re-

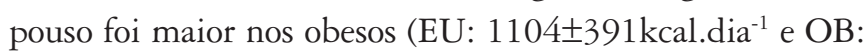
$\left.1489 \pm 282 \mathrm{kcal} \mathrm{dia}^{-1} ; p \leq 0,01\right)$. Esses valores se correlacionaram positiva e significativamente com o peso corporal total $(\mathrm{r}=0,62 ; p \leq 0,01)$, com a massa magra $(\mathrm{r}=0,47 ; p \leq 0,05)$ e com a gordura corporal $(\mathrm{r}=0,61 ; p \leq 0,01) . \mathrm{O} \mathrm{VO}_{2 \text { pico }}\left(\mathrm{mL} \cdot \mathrm{kg}^{-1}\right.$. $\mathrm{min}^{-1}$ ) apresentou correlação positiva e significativa com o percentual de massa magra $(r=0,66 ; p \leq 0,01)$ e correlação negativa e significativa com a massa gorda $(r=-0,66 ; p \leq 0,01)$.

A Tabela 2 apresenta dados de ingestão e gasto de energia pelos grupos. Os três métodos são discordantes entre si e o gasto energético diário é maior no grupo OB pelos três métodos utilizados. A diferença entre ingestão e gasto ou balanço energético (BE) é negativa em EU somente quando 
Tabela 1 - Variáveis antropométricas de composição corporal e de consumo de oxigênio nos grupos estudados

\begin{tabular}{|c|c|c|c|c|c|}
\hline & \multicolumn{2}{|c|}{ Eutróficos $(n=11)$} & \multicolumn{2}{|c|}{ Obesos $(n=12)$} & \multirow{2}{*}{ Valor de $p$} \\
\hline & Média \pm DP & Percentil & Média $\pm D P$ & Percentil & \\
\hline Peso corporal $(\mathrm{kg})$ & $64,7 \pm 8,8$ & P50 & $91,6 \pm 13,1$ & P97 & $<0,001$ \\
\hline Estatura $(\mathrm{cm})$ & $173,4 \pm 4,6$ & P25 & $170,0 \pm 6,8$ & P50 & 0,18 \\
\hline IMC $\left(\mathrm{kg} \cdot \mathrm{m}^{-2}\right)$ & $21,6 \pm 2,8$ & P50 & $31,6 \pm 3,4$ & P95 & $<0,001$ \\
\hline Circunferência da cintura $(\mathrm{cm})$ & $75,6 \pm 8,9$ & $<\mathrm{P} 80$ & $102,6 \pm 9,0$ & $>\mathrm{P} 80$ & $<0,001$ \\
\hline Massa magra corporal (kg) & $56,1 \pm 6,1$ & P25 & $63,0 \pm 9,5$ & P50 & 0,05 \\
\hline Massa magra corporal (\%) & $86,6 \pm 5,8$ & ND & $68,8 \pm 3,9$ & ND & $<0,001$ \\
\hline Massa gorda corporal $(\mathrm{kg})$ & $8,5 \pm 4,1$ & P25 & $28,6 \pm 5,6$ & P95 & $<0,001$ \\
\hline Massa gorda corporal (\%) & $12,8 \pm 4,7$ & P25 & $31,2 \pm 3,9$ & P95 & $<0,001$ \\
\hline 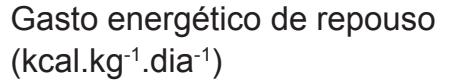 & $17,0 \pm 5,1$ & - & $16,4 \pm 2,9$ & - & 0,7 \\
\hline $\mathrm{VO}_{2}$ pico $\left(\mathrm{mL} \cdot \mathrm{kg}^{-1} \cdot \mathrm{dia}^{-1}\right)$ & $50,82 \pm 6,7$ & - & $38,4 \pm 8,2$ & - & $<0,001$ \\
\hline
\end{tabular}

IMC: índice de massa corporal; ND: não determinado, por não existirem estudos de referência.

Tabela 2 - Variáveis relativas à ingestão e gasto de energia diario (GED), obtidos a partir de três diferentes métodos

\begin{tabular}{lll}
\hline & \multicolumn{1}{c}{ Eutróficos } & \multicolumn{1}{c}{ Obesos } \\
\hline GED1, diário de atividade física (kcal) & $2.752,7 \pm 701,8^{\text {*\# }}$ & $3.622,9 \pm 643,7^{\text {*\# }}$ \\
GED2, fórmulas preditivas (kcal) & $3.261,9 \pm 353,9 \mathrm{~b}^{\text {*\# }}$ & $4.019,3 \pm 672,3^{\text {*\# }}$ \\
GED3, calorimetria indireta $(\mathrm{kcal})$ & $2.077,7 \pm 766,7^{\text {*\# }}$ & $2.640,1 \pm 512,3^{\star \#}$ \\
Ingestão de energia relatada $(\mathrm{kcal})$ & $2.620,0 \pm 701,0^{2}$ & $3.071,0 \pm 809,4^{1,2}$ \\
\hline
\end{tabular}

*: diferença entre eutróficos e obesos $(p<0,05)$; \#: diferença entre os métodos de determinação; 1: diferença em relação ao GED1 obtido pelos diários de atividade física; 2: diferença em relação ao GED2, obtido pelas fórmulas preditivas.

determinado por equações preditivas. Para o grupo $\mathrm{OB}$, o $\mathrm{BE}$ foi negativo quando estimado por recordatórios de atividade física e por equações preditivas.

A Tabela 3 apresenta a descrição quantitativa da dieta. Para ambos os grupos, o consumo de carboidratos, em valores absolutos (g) foi acima do proposto pelas DRIs ${ }^{(11)} \mathrm{e}$, percentualmente, ficou no limite inferior dessa proposta. $\mathrm{O}$ percentual de lipídeos ficou no limite superior do intervalo sugerido para ambos os grupos. A ingestão de proteínas foi acima do sugerido, tanto em g.dia ${ }^{-1}$ quanto em g.kg peso corporal ${ }^{-1} \cdot \mathrm{dia}^{-1}$, para ambos os grupos. A ingestão de cálcio foi muito inferior aos valores de referência para ambos os grupos, assim como os valores de fibras alimentares. Nenhum dos valores obtidos indicou diferença entre os grupos.

A Tabela 4 apresenta o consumo das porções dos grupos de alimentos e confronta os valores sugeridos pelo Guia Alimentar Brasileiro. A despeito da grande dispersão dos dados, os EU apresentaram consumo adequado de cereais, raízes e tubérculos, enquanto os $\mathrm{OB}$ apresentaram um baixo consumo desses alimentos. Tanto os OB quanto os EU apresentaram consumo baixo de hortaliças. O consumo de frutas foi baixo por ambos os grupos, embora o EU tenha apresentado consumo significativamente maior. Nenhum dos grupos apresentou ingestão adequada de leite e derivados. O consumo de leguminosas foi muito próximo ao sugerido pelo guia no grupo EU e foi significativamente maior que o grupo OB. Ambos os grupos apresentaram um alto consumo de carnes e ovos, sem diferença entre EU e OB. O consumo de alimentos fonte de gorduras foi elevado em ambos os grupos e foi significativamente maior no grupo OB. O grupo EU apresentou um consumo maior de açúcares e doces.

No que diz respeito a aspectos qualitativos das dietas dos adolescentes, nenhum adolescente do grupo EU consumiu o café da manhã regularmente (nos três dias analisados), enquanto no grupo OB apenas cinco adolescentes o fizeram; o número mediano de refeições realizadas pelos grupos foi 3 no grupo EU e 1 no grupo $\mathrm{OB}$; com relação à variedade da dieta, ambos os grupos consumiram o valor mediano de 10 diferentes tipos de alimentos nos 3 dias analisados; o grupo EU consumiu uma média de $402 \mathrm{~mL}$ de refrigerantes por dia, enquanto o grupo OB consumiu uma média de $265 \mathrm{~mL}$. Esses valores não foram diferentes entre os grupos. 
Tabela 3 - Análise quantitativa da dieta realizada pelos adolescentes

\begin{tabular}{lccccc}
\hline & & Eutróficos $\mathbf{( n = 1 1 )}$ & Obesos $(\mathbf{n}=\mathbf{1 2})$ & DRI & Valor de $\boldsymbol{p}$ \\
\hline Carboidratos & $\mathrm{g}$ & $297 \pm 71$ & $353 \pm 86$ & 130 & 0,10 \\
& $\mathrm{~g} \cdot \mathrm{kg}^{-1}$ & $4,6 \pm 1,3$ & $3,93 \pm 1,05$ & $\mathrm{ND}$ & 0,16 \\
Lipídeos & $\%$ & $46 \pm 6$ & $46 \pm 9$ & $45-65$ & 0,81 \\
& $\mathrm{~g}$ & $98(55-144)$ & $110(86-264)$ & $\mathrm{ND}$ & 0,58 \\
Proteínas & $\%$ & $35 \pm 5$ & $36 \pm 8$ & $25-35$ & 0,87 \\
& $\mathrm{~g}$ & $91(53-272)$ & $105(87-206)$ & 34 & 0,32 \\
Cálcio & $\mathrm{g} \cdot \mathrm{kg}^{-1}$ & $1,6(1,0-2,2)$ & $1,19(0,82-2,85)$ & 0,73 & 0,09 \\
Ferro & $\%$ & $17 \pm 4$ & $16 \pm 2$ & $10-35$ & 0,39 \\
Fibras alimentares & $\mathrm{mg}$ & $327(204-1051)$ & $451(213-947)$ & 1300 & 0,20 \\
\hline
\end{tabular}

ND: valores não determinados pelas DRIs (dietary reference intakes) ${ }^{(11)}$.

Tabela 4 - Análise qualitativa e semiquantitativa da dieta realizada pelo grupo de adolescentes

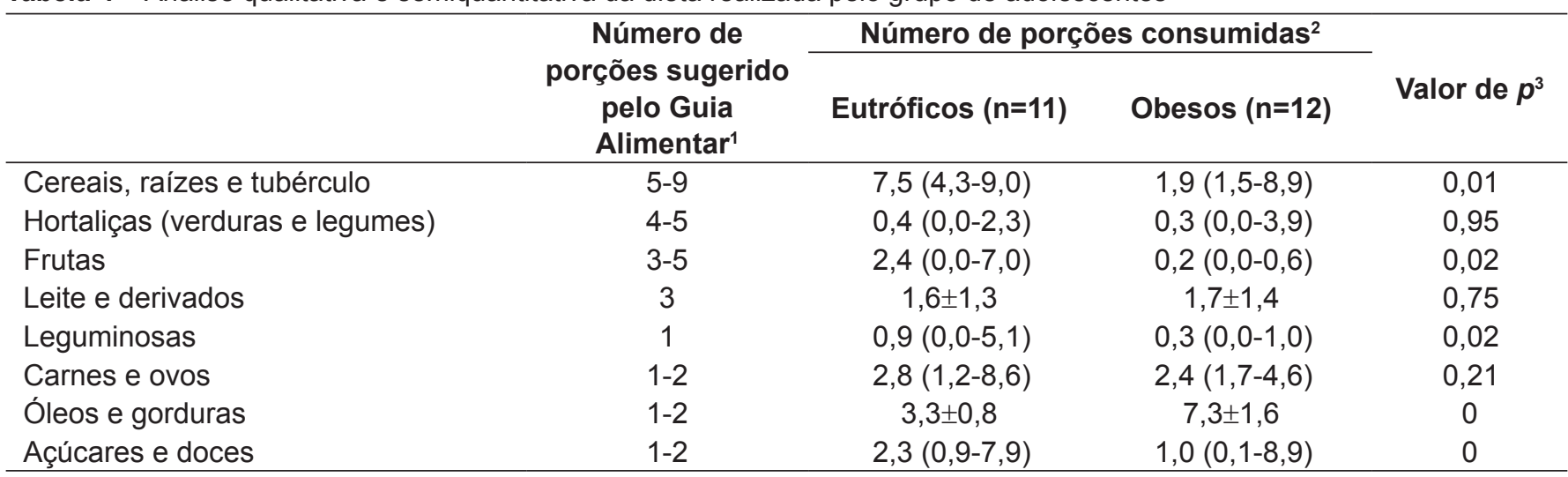

1: Guia Alimentar para a População Brasileira(12); 2: os valores que não apresentaram distribuição normal estão apresentados em forma de mediana, valores mínimos e máximos; os que apresentaram distribuição normal estão em forma de média e desvio padrão. 3: todos os valores foram comparados pelo teste $\mathrm{U}$ de Mann-Witney, para dados não paramétricos.

\section{Discussão}

Entre os principais achados, destacam-se: o gasto energético diário dos obesos foi maior, porém a ingestão alimentar foi semelhante à dos eutróficos; não foram encontradas diferenças no gasto energético de repouso entre os grupos; os valores de potência aeróbia foram mais elevados nos eutróficos; observou-se uma discrepância na análise do gasto energético diário, quando se adotou diferentes métodos; embora os obesos tenham apresentado baixo consumo de alimentos considerados saudáveis e um consumo aumentado de alimentos fontes de gorduras, a dieta de ambos os grupos não pode ser considerada saudável.

Não houve diferença no gasto energético em repouso entre os grupos. Isso é compatível com estudos recentes que descartam hipóteses mais antigas, que atribuíam ao menor gasto energético de repouso o fato de algumas pessoas serem obesas. Atualmente, é consensual afirmar que indivíduos obe- sos gastam mais energia para 'carregar' seu peso corporal ${ }^{(3)}$. Por outro lado, o grupo de obesos apresentou menor potência aeróbia, o que significa menor capacidade muscular de captar e utilizar oxigênio. Desta forma, a correlação entre $\mathrm{VO}_{\text {2pico }}$ e a massa magra corporal encontrada é esperada. Isso permite hipotetizar que, a despeito da maior massa magra nos obesos, esse tecido poderia apresentar qualidade diferente do grupo eutrófico. Marks e Rippe ${ }^{(18)}$ destacaram que o músculo esquelético em indivíduos obesos é caracterizado por sua baixa densidade, menor concentração de mitocôndrias e consequentemente menor capacidade de trabalho.

No presente estudo, procurou-se identificar variáveis dietéticas relacionadas à obesidade. Essa relação (alimentação e obesidade) tem sido objeto de uma série de investigações, sem contudo existir um consenso ${ }^{(19,20)}$ : alguns estudos encontram relação significativa ${ }^{(20)}$ e outros não ${ }^{(21,22)}$. Observou-se tendência, no grupo EU, de maior ingestão de proteínas $(p=0,09)$. Alguns autores têm investigado benefício do aumento de 
proteínas da dieta com relação à obesidade, seja pela maior termogênese resultante ${ }^{(22)}$ ou pelo maior estímulo à síntese proteica $^{(23)}$. Por sua vez, o grupo obeso apontou preferência por alimentos fontes de lipídeos, ingestão esta que não foi ratificada pelo cálculo quantitativo da dieta. $\mathrm{O}$ pequeno número de indivíduos estudados pode ter sido a razão para essa discrepância.

O consumo do café da manhã não foi um hábito comum nos adolescentes. Estudos associam esse fato com a obesidade, por várias razões ${ }^{(24-26)}$. O consumo do café da manhã exerce controle sobre fome e apetite e sobre alguns hormônios; consequentemente, ocorre controle glicêmico e lipídico. Os alimentos normalmente consumidos no café da manhã (frutas, laticínios e cereais integrais) são fontes de fibras alimentares (controlam a absorção de alimentos e consequentemente os mecanismos de fome e saciedade). Alimentos fonte de cálcio também têm sido relacionados ao controle da obesidade, por mecanismos relacionados à termogênese e/ou síntese de triacilgliceróis no adipócito ${ }^{(26)}$. O consumo de cálcio e fibras foi baixo para ambos os grupos, o que pode guardar, portanto, relação com a baixa incidência do café da manhã nos períodos avaliados.

Nem todos os estudos têm encontrado relação direta entre o consumo do café da manhã e obesidade. Um fator de confusão associado a isso pode ser o baixo número de refeições realizadas durante o dia, fato este também constatado no presente estudo ${ }^{(27)}$. Maior número de refeições significa menor estímulo insulinêmico a cada refeição (pressupondo-se que a carga energética de cada refeição será menor), o que significa menor desvio para a lipogênese ${ }^{(28-30)}$.

Com relação ao gasto energético, este foi maior no grupo obeso com os três métodos utilizados. A estimativa por fórmulas preditivas resultou em maiores valores de GED, em ambos os grupos. Cruz et al ${ }^{(31)}$ observaram superestimativa do gasto energético por fórmulas preditivas. Por sua vez, Neilson et $a l^{(32)}$, em um estudo de metanálise, mostraram ser comum o super-relato de atividade física quando se utiliza questionários. Comparando a ingestão de energia com o gasto obtido pelos recordatórios de atividade física, verificou-se um balanço energético negativo em ambos os grupos.

De acordo com a $\mathrm{FAO} / \mathrm{WHO} / \mathrm{UNU}^{(16)}$, se os indivíduos se encontram "na média em estado firme, com a composição corporal e o nível de atividade física apropriados", não se pode considerar o quadro indicativo de subconsumo. Em se tratando de obesos, não foram observados sinais de perda de peso nos últimos meses. Portanto, a hipótese de balanço negativo é pouco provável. Por sua vez, é bem abordada na literatura a limitação dos métodos para avaliar o consumo alimentar em obesos, o que é descrito como sub-relato. Alguns autores afirmam que indivíduos tendem a relatar ingestas similares ao esperado pela população em geral ou para indivíduos não-obesos. Ainda, adolescentes tendem a subnotificar sua dieta ou a relatar consumo de alimentos com menor valor nutricional, inconsciente ou conscientemente ${ }^{(19,33,34)}$. O sub-relato seletivo pode ser consequência do maior nível de conhecimento sobre alimentação, visto que os adolescentes frequentam a escola e, portanto, recebem informações sobre dieta saudável.

$\mathrm{Na}$ análise de balanço energético por calorimetria indireta, ambos os grupos apresentaram balanço energético positivo, o que é mais coerente com as características do presente estudo. Neste caso, o grupo eutrófico pode ser considerado como em risco de desenvolver sobrepeso.

Qualquer que seja o tipo de análise - de balanço energético, de qualidade dos alimentos ou de grupos de alimentos -, observam-se, tanto nos obesos quanto nos eutróficos, hábitos alimentares favoráveis ao desenvolvimento de obesidade e doenças crônicas. A hipótese de que os obesos têm uma alimentação menos saudável não pode ser comprovada. Se os hábitos alimentares inadequados neste estudo não se limitaram apenas aos obesos, perguntase: esses hábitos são determinantes ou consequência da obesidade? Fatores relacionados à genética, ao estilo de vida e à dieta contribuem diferentemente para a epidemia da obesidade e, portanto, intervenções dietéticas devem modificar parcialmente a 'história natural' da obesidade. O fenótipo da obesidade é a expressão final em indivíduos predispostos geneticamente e, portanto, é o resultado da interação entre genes e fatores ambientais. É imperativo criar 'ambientes favoráveis' para concretizar um padrão alimentar apropriado, no intuito de prevenir ou controlar a obesidade e as doenças relacionadas.

Finalmente, é importante destacar algumas limitações do presente estudo: a amostra foi selecionada a partir de um número pequeno de escolas e foram incluídos aqueles indivíduos que se voluntariaram a participar da análise. O grande número de variáveis analisadas e, portanto, a grande dedicação exigida dos sujeitos, resultou em pequeno tamanho amostral, o que impossibilita a generalização dos resultados para a população em geral. É importante ainda lembrar que somente estudos com caráter epidemiológico permitiriam conclusões mais concretas relacionadas à ingestão alimentar e obesidade. 
Portanto, com base nas condições experimentais e nos objetivos do presente estudo, conclui-se que, independentemente do fato de serem obesos ou não, os adolescentes apresentaram um padrão alimentar relacionado ao risco de obesidade e doenças crônicas, sendo urgente a busca de estratégias de educação nutricional específicas para esse grupo etário. Não foram encon- tradas diferenças no gasto energético de repouso que pudessem justificar a presença de obesidade. Por sua vez, adolescentes eutróficos apresentaram maior potência aeróbia, o que confere a esses indivíduos melhor condicionamento cardiovascular. Mais estudos são necessários para identificar melhores métodos e técnicas para avaliar o gasto energético nesses indivíduos.

\section{Referências bibliográficas}

1. Conti MA, Frutuoso MF, Gambardella AM. Obesity and body dissatisfaction amongst adolescents. Rev Nutr 2005;18:491-7.

2. Mondini L, Monteiro CA. Relevance of under and overnutrition in different social classes: methods and application to the Brazilian population. Rev Bras Epidemiol 1998;1:28-39.

3. Melo CM, Tirapegui J, Ribeiro SML. Gasto energético corporal: conceitos, formas de avaliação e sua relação com a obesidade. Arq Bras Endrocrinol Metab 2008;52:452-64.

4. Tanner JM. Growth at adolescence. $2^{\text {nd }}$ ed. Oxford: Blackwell, 1962.

5. Pardini R, Matsudo S, Araujo T, Matsudo V, Andrade E, Braggion G et al. Validation of the International Physical Activity Questionaire (IPAQ version 6): pilot study in Brazilian young adults. Rev Bras Cien Mov 2001;9:45-51.

6. Must A, Dallal GE, Dietz WH. Reference data for obesity: $85^{\text {th }}$ and $95^{\text {th }}$ percentiles of body mass index $\left(\mathrm{wt}_{\mathrm{h}} / \mathrm{ht}^{2}\right)$ and triceps skinfold thickness. Am J Clin Nutr 1991;53:839-46.

7. World Health Organization. Physical status: the use and interpretation of anthropometry: Report of a WHO Expert Committee. Geneva: WHO; 1995.

8. Taylor RW, Jones IE, Williams SM, Goulding A. Evaluation of waist circumference, waist-to-hip ratio, and the conicity index as screening tools for high trunk fat mass, as measured by dual-energy X-ray absorptiometry, in children aged 3-19 y. Am J Clin Nutr 2000;72:490-5.

9. Pietrobelli A, Boner AL, Tatò L. Adipose tissue and metabolic effects: new insight into measurements. Int J Obes (Lond) 2005;29:S97-100.

10. Pichard C, Kyle UG, Bracco D, Slosman DO, Morabia A, Schutz Y. Reference values of fat-free and fat masses by bioelectrical impedance analysis in 3393 healthy subjects. Nutrition 2000;16:245-54

11. Institute of Medicine, Food and Nutrition Board. Dietary reference intakes for energy, carbohydrates, fiber, fat, protein and amino acids (macronutrients). Washington, DC: National Academy Press; 2002.

12. Brasil - Ministério da Saúde. Guia Alimentar para a população brasileira: promovendo a alimentação saudável. Série A. Normas e Manuais Técnicos. Brasilia: Ministério da Saúde, 2006.

13. Fransen HP, Ocké MC. Indices of diet quality. Curr Opin Clin Nutr Metab Care 2008;11:559-65.

14. Lennernäs M, Andersson I. Food-based classification of eating episodes (FBCE). Appetite 1999;32:53-65.

15. Bouchard C, Tremblay A, Leblanc C, Lortie G, Savard R, Thériault G. A method to assess energy expenditure in children and adults. Am J Clin Nutr 1983; 37:461-7.

16. Food and Agriculture Organization, World Health Organization, United Nations University. Energy and protein requirements: WHO Technical Report Series 724, Geneva: WHO, 1985.

17. Jéquier $E$, Schutz $Y S$. Long-term measurements of energy expenditure in humans using a respiration chamber. Am J Clin Nutr 1983;38:989-98.

18. Marks BL, Rippe JM. The importance of fat free mass maintenance in weight loss programmes. Sports Med 1996;22:273-81.

19. Livingstone MB, Robson PJ. Measurement of dietary intake in children. Proc Nutr Soc 2000;59:279-93.

20. Gillis LJ, Kennedy LC, Gillis AM, Bar-Or O. Relationship between juvenile obesity, dietary energy and fat intake and physical activity. Int J Obes Relat Metab Disord 2002;26:458-63.

21. Atkin LM, Davies PS. Diet composition and body composition in preschool children. Am J Clin Nutr 2000;72:15-21.

22. Petzke KJ, Klaus S. Reduced postprandial energy expenditure and increased exogenous fat oxidation in young woman after ingestion of test meals with low protein content. Nutr Metab (Lond) 2008;17:5-25.

23. Layman DK, Evans EM, Erickson D, Seyler J, Weber J, Bagshaw D et al. A moderate-protein diet produces sustained weight loss and long-term changes in body composition and blood lipids in obese adults. J Nutr 2009;139:514-21.

24. Bellisle F, Rolland-Cachera MF, Kellogg Scientific Advisory Committee. Three consecutive $(1993,1995,1997)$ surveys of food intake, nutritional attitudes and knowledge, and lifestyle in 1000 French children, aged 9-11 years. J Hum Nutr Diet 2007;20:241-51.

25. Giovannini M, Verduci E, Scaglioni S, Salvatici E, Bonza M, Riva E et al. Breakfast: a good habit, not a repetitive custom. J Int Med Rec 2008;36:613-24.

26. Zemel MB. Mechanisms of dairy modulation of adiposity. J Nutr 2003;133: 252S-6.

27. Toschke AM, Kuchenhoff $H$, Koletzko B, von Kries R. Meal frequency and childhood obesity. Obes Res 2005;13:1932-8.

28. Farshchi HR, Taylor MA, MacDonald IA. Beneficial metabolic effects of regular meal frequency on dietary thermogenesis, insulin sensitivity, and fasting lipid profiles in healthy obese women. Am J Clin Nutr 2005;81:16-24.

29. Ma Y, Bertone ER, Stanek EJ 3rd, Reed GW, Herbert JR, Cohen NL et al. Association between eating patterns and obesity in a free-living US adult population. Am J Epidemiol 2003;158:85-92.

30. Rolland-Cachera MF, Bellisle F. Child and adolescent obesity. Causes and consequences, prevention and management. In: Burniat W, Cole TJ, Lissau I, Poskit EM, editors. Child and adolescent obesity. Cambridge: Cambridge University Press, 2002.

31. Cruz CM, da Silva AF, dos Anjos LA. Basal metabolic rate is overestimated by predictive equation in college-age women of Rio de Janeiro, Brazil. Arch Latinoam Nutr 1999;49:232-7.

32. Neilson HK, Robson PJ, Friedenreich CM, Csizmadi I. Estimating activity energy expenditure: how valid are physical activity questionnaires? Am J Clin Nutr 2008;87:279-91.

33. Bandini LG, Shoeller DA, Cyr HN, Dietz WH. Validity of reported energy intake in obese and nonobese adolescents. Am J Clin Nutr 1990;52:421-5.

34. Krebs-Smith SM, Graubard BI, Kahle LL, Subar AF, Cleveland LE, BallardBarbash R. Low energy reporters vs others: a comparison of reported food intakes. Eur J Clin Nutr 2000;54:281-7. 